\title{
Fuzzy multiwavelet denoising on an ECG signal
}

\author{
Charlotte Yuk-Fan Ho, Bingo Wing-Kuen Ling, Thomas Pak-Lin Wong, Albert \\ Yick-Po Chan, and Peter Kwong-Shun Tam
}

\begin{abstract}
Since different multiwavelets, pre- and post-filters have different impulse and frequency responses characteristics, different multiwavelets, pre- and post-filters should be selected, integrated and applied at different noise levels if a signal is corrupted by an additive white Gaussian noise (AWGN). In this letter, some fuzzy rules on selecting and integrating different multiwavelets, pre- and post-filters together are proposed. These fuzzy rules are setup based on the training results of the denoising performances of applying different multiwavelets, pre- and post-filters at different noise levels. When a new electrocardiogram (ECG) signal is applied, the appropriate multiwavelets, pre- and post-filters are selected and integrated based on fuzzy rules and the noise level of the signal. A hard thresholding is applied on the multiwavelet coefficients. According to an extensive simulation, we found that our proposed fuzzy rule-based multiwavelet denoising algorithm achieves 30\% improvement compared to the traditional multiwavelet denoising algorithms.
\end{abstract}

\section{INTRODUCTION}

The multiwavelet theory has already proved its efficiency for the signal denoising problem. However, what multiwavelets, pre- and post-filters should be used? Do the selections depend on the noise levels? The aim of this letter is to propose a method to select and integrate different multiwavelets, pre- and post-filters together to 
obtain sub-optimal results for the ECG denoising problem.

\section{Proposed multiwavelet fuzzy denoising algorithm}

The block diagram of our proposed multiwavelet fuzzy denoising system comprises with six blocks as in Figure 1. Since two types of Chui Lian (CL) multiwavelets [1, p.289, p.295] and Qingtnag Jiang (QJ) multiwavelet [2] are the most widely used in the signal processing area, we choose these three multiwavelets. Besides, thirty-five commonly used pre- and post-filters, including symlets, coiflets, Daubenchies and biorthogonal filters, are also used, as listed in Table 1.

The fuzzy rules are established based on the following methods: A set of input-output relations that describes the denoising performances of applying different multiwavelets, pre- and post-filters at different noise levels are obtained based on training samples in the database [3], where the denoising performances are calculated based on the mean square error (MSE) criterion. A TS fuzzy model is established based on trapezoidal fuzzy membership functions described in Figures 2 and 3. The fuzzy rules are summarized in Table 2 .

A hard thresholding based on the Donoho approach is applied on the multiwavelet coefficients [4].

\section{Experimental results}

The ECG signals used in the experiment are from the Beth Israel Deaconess Medical Center at Boston [3]. In order to demonstrate the sub-optimality of different multiwavelets, pre- and post-filters, computer simulations were performed to recover signals of 1000 points embedded in the zero mean AWGN at various noise levels. 
Figure 4 shows the average of the MSE of signals in [3] for the fuzzy and non-fuzzy methods. The non-fuzzy method is described as univariate soft thresholding in GHM multiwavelet domain [5]. Compared the result of two methods, the fuzzy systems do provide better denoising performances as expected with consistently about $30 \%$ MSE improvement.

\section{Conclusions}

This letter proposes an intelligent based method for selecting and integrating different multiwavelets, pre- and post-filters together at different noise levels for the ECG signal denoising problem. According to our extensive simulations, we found that our proposed fuzzy system gives an excellent performance.

\section{ACKNOWLEDGEMENT}

The work obtained in this letter was supported partially by a studentship and partially by a research grant (G-YD26) of The Hong Kong Polytechnic University.

\section{REFERENCES}

[1] Charles K. Chui and Jian-ao Lian, "A study of orthonormal multi-wavelets," Applied Numerical Mathematics, vol. 20, pp. 273-298, 1996.

[2] Qingtang Jiang, "On the design of multifilter banks and orthonormal multiwavelet bases," IEEE Transactions on Signal Processing, vol. 46, no. 12, pp. 3292-3303, December 1998.

[3] http://www.physionet.org/physiobank/database/mitdb/

[4] David L. Donoho, "De-noising by soft-thresholding," IEEE Transactions on 
Information Theory, vol. 41, no. 3, pp. 613-627, May 1995.

[5] Tien D. Bui and Guangyi Chen, "Translation-invariant denoising using multiwavelets," IEEE Transactions on Signal Processing, vol. 46, no. 12, pp. 3414-3420, December 1998. 


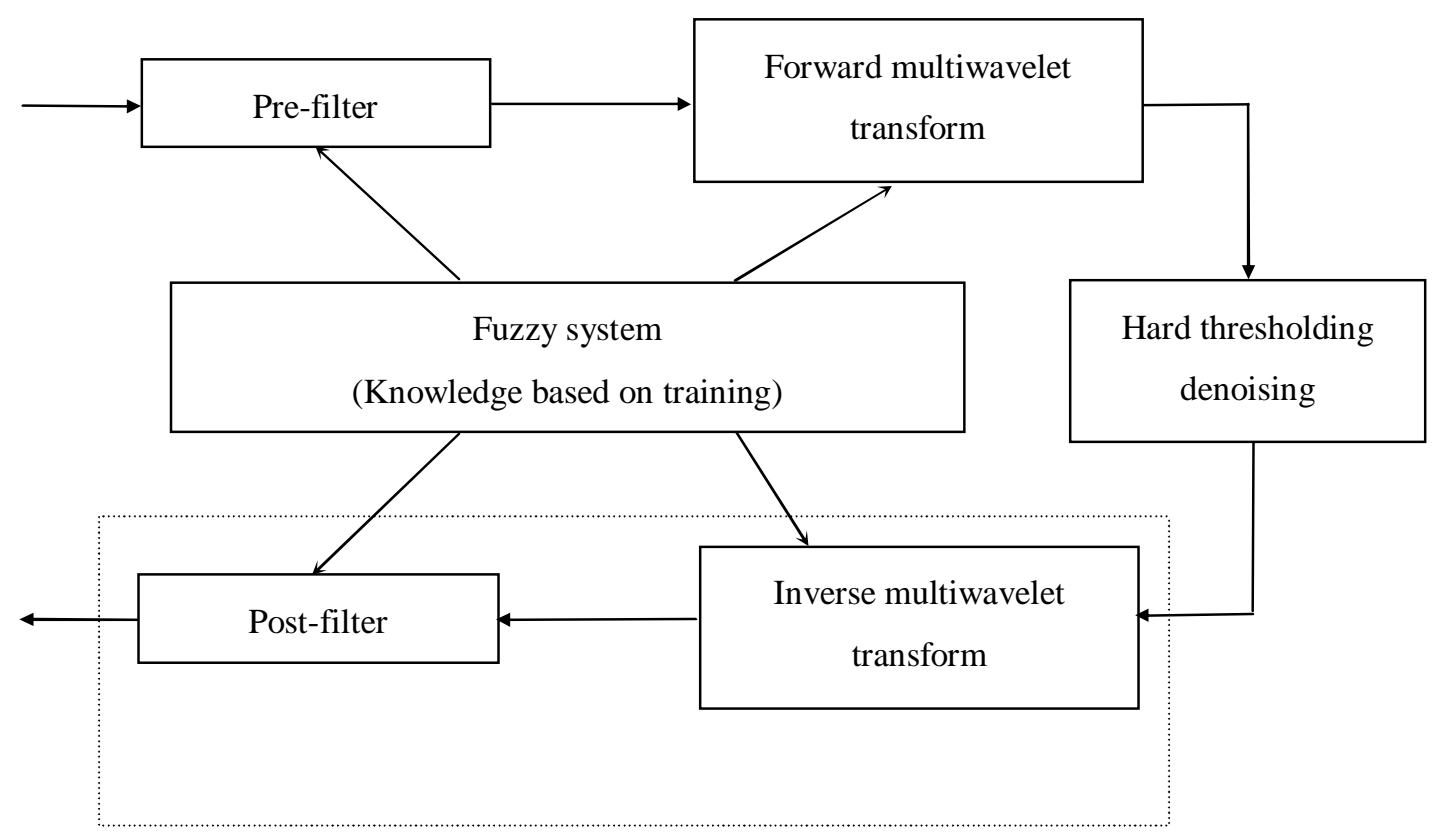

Figure 1: Block diagram of multiwavelet fuzzy denoising system

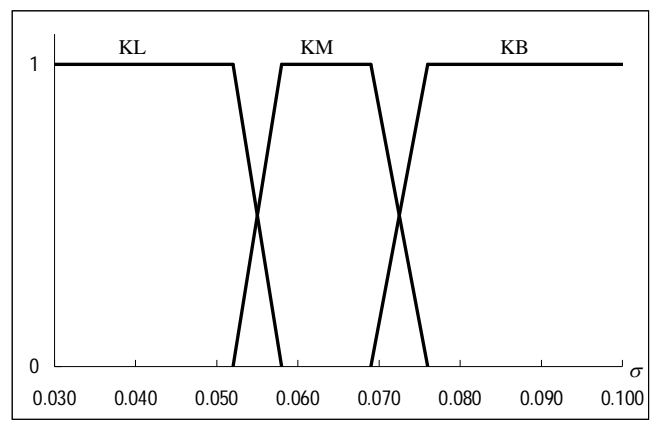

Figure 2: Membership functions of multiwavelet kernels

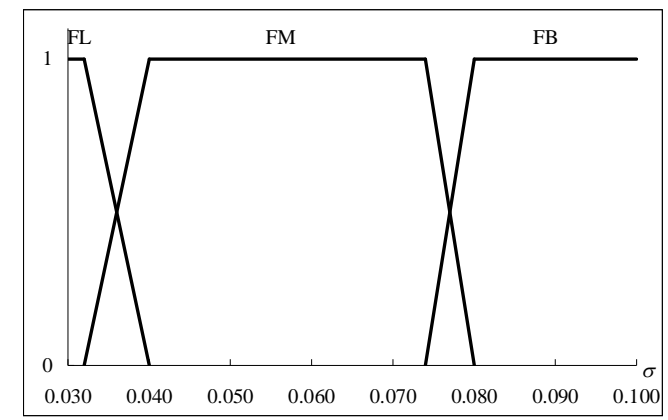

Figure 3: Membership functions of pre- and post-filters 


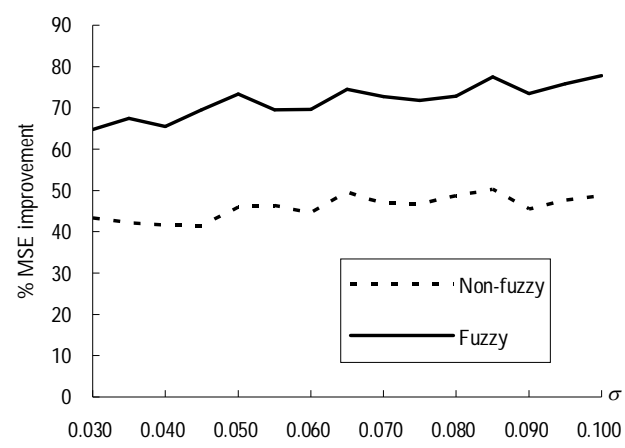

Figure 4: Comparison of denoising performance

\begin{tabular}{|c|c|c|}
\cline { 2 - 3 } \multicolumn{1}{c|}{} & \multicolumn{2}{|c|}{ Name } \\
\hline \multirow{2}{*}{$\begin{array}{c}\text { Multiwavelet } \\
\text { kernels }\end{array}$} & \multicolumn{2}{|c|}{ Chiu Lian [1, p.289, p.295] } \\
\cline { 2 - 3 } & Symlets & Qingtang Jiang [2] \\
\cline { 2 - 3 } Pre- and & Coiflets & 1 to 5 \\
\cline { 2 - 3 } post-filters & Daubenchies & 1 to 8 \\
\cline { 2 - 3 } & Biorthogonal & $\begin{array}{r}1.1,1.3,1.5,2.2,2.4,2.6,2.8,3.1,3.3, \\
\end{array}$ \\
& & $3.5,3.7,3.9,4.4,5.5$ and 6.8 \\
\hline
\end{tabular}

Table 1: Multiwavelet kernels, pre- and post-filters

\begin{tabular}{|l|l|}
\hline Rules & Kernels \\
\hline If KL, & then CL2 or QJ. \\
\hline If KM, & then CL2. \\
\hline If KB, & then QJ. \\
\hline Rules & Filters \\
\hline If FL, & then Symlets 2. \\
\hline If FM, & then Biorthogonal 3.3. \\
\hline If FB, & then Biorthogonal 3.1. \\
\hline
\end{tabular}

Table 2: Sets of fuzzy rules 